\title{
ESTUDO DO COMPORTAMENTO FOTOCRÓMICO DE UM NAFTOPIRANO: UMA EXPERIÊNCIA SIMPLES ILUSTRATIVA DO FOTOCROMISMO
}

Paulo J. Coelho

Departamento de Química, Universidade de Trás-os-Montes e Alto Douro, 5001-911 Vila Real, Portugal

Recebido em 24/5/05; aceito em 5/9/05; publicado na web em 16/2/06

\begin{abstract}
STUDY OF THE PHOTOCHROMIC BEHAVIOUR OF A NAPHTHOPYRAN: A SIMPLE EXPERIMENT ILLUSTRATIVE OF THE PHOTOCHROMIC PHENOMENON. An easy experiment on the synthesis of 3,3-diphenyl-3H-naphtho[2,1-b]pyran and characterization of its photochromic behaviour is reported. Upon irradiation of an uncoloured toluene solution of 3,3-diphenyl$3 H$-naphtho[2,1-b]pyran with near UV light, an intense yellow colouration is produced due to the formation of two coloured open forms. When the irradiation source is removed the coloured open forms return to the uncoloured state. The analysis of the absorbance decay with time allows the determination of the discoloration rate constants of each open form. The reversibility of the photochromic phenomenon, well demonstrated by the exposure of the solution to sunlight for a few seconds, is particularly attractive to the students.
\end{abstract}

Keywords: spectrokinetics; naphthopyran; photochromism.

\section{INTRODUÇÃO}

Algumas moléculas quando irradiadas com luz ultravioleta alteram a sua cor de um modo reversível. Este fenómeno, designado por fotocromismo, foi descoberto no século XIX mas só viria a despertar o interesse alargado da comunidade científica no final do século XX quando se intensificou a sua aplicação prática. O termo fotocromismo designa a transformação química reversível entre duas espécies com espectros de absorção diferentes promovida, pelo menos numa direcção, por radiação electromagnética, geralmente UV. A transformação inversa pode ser térmica ou também fotoquímica ${ }^{1}$.

Existem diversas classes de compostos fotocrómicos geralmente classificados de acordo com o tipo de reacção inversa. Os naftopiranos, espirooxazinas e espiropiranos são activados por luz UV e revertem ao estado inicial termicamente, enquanto que os diariletenos e as fulgidas sendo activados por radiação UV, só revertem ao estado inicial por irradiação com luz visível de comprimento de onda variável ${ }^{2}$.

As espécies químicas envolvidas neste processo podem diferir não só no seu espectro de absorção como também noutras propriedades físico-químicas como a condutividade eléctrica, constante dieléctrica, índice de refracção, etc. Nos últimos anos têm sido desenvolvidas diversas aplicações destes compostos que aproveitam directamente a variação reversível de cor, tais como, lentes oftálmicas fotocrómicas ${ }^{3}$, tintas, vernizes e corantes fotocrómicos. Outras aplicações, ainda em desenvolvimento, aproveitam a variação reversível de outras propriedades físico-químicas. São exemplos disso a utilização de sistemas fotocrómicos em memórias ópticas e outros sistemas optoelectrónicos ${ }^{2}$.

\section{FOTOCROMISMO NOS NAFTOPIRANOS}

Os naftopiranos são uma das classes de compostos fotocrómicos mais estudadas nos últimos 20 anos. Estas moléculas são geralmente incolores em solução, apresentando uma banda de absorção intensa na região do UV próximo, entre 300 e 400 nm. A irradiação

*e-mail: pcoelho@utad.pt destas moléculas, em solução ou incorporadas em matrizes poliméricas, com luz UV promove a quebra da ligação C-O com abertura do ciclo pirânico e formação de um conjunto de formas abertas coradas com um sistema de conjugação estendido (Esquema 1). Quando a fonte de irradiação é removida o sistema reverte ao estado inicial incolor em tempo variável (desde alguns segundos até vários dias) geralmente através de um processo térmico ${ }^{4}$.

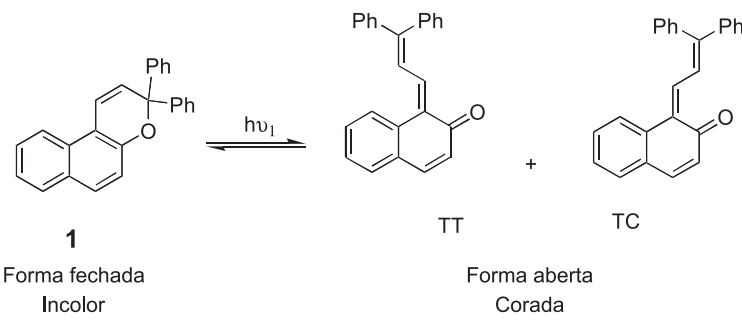

Esquema 1. Fotocromismo nos naftopiranos

O 3,3-difenil-3H-nafto[2,1-b]pirano 1 é um dos naftopiranos mais estudados. As soluções deste composto são incolores mas quando irradiadas por luz solar adquirem uma intensa coloração amarela. Esta alteração do espectro de absorção resulta do aumento da conjugação dos electrões $\pi$ : enquanto que na forma fechada não há conjugação entre os substituintes fenilo do anel pirânico e o anel naftalénico, devido à existência de um átomo de carbono $\mathrm{sp}^{3}$ entre eles, nas formas abertas essa conjugação estende-se desde o anel naftalénico até a um dos grupos fenilo. Quer o comprimento de onda de absorção máxima quer a intensidade de cor obtida após irradiação podem ser modificados mediante alterações estruturais na molécula, que envolvem geralmente a introdução de substituintes dadores de electrões e a fusão de anéis aromáticos ${ }^{4-8}$. Actualmente é possível obter qualquer coloração usando apenas naftopiranos ${ }^{3}$.

A irradiação dos naftopiranos com luz UV conduz à formação maioritária de duas formas abertas coloridas isoméricas, designadas por TT e TC. Estas espécies não são estáveis e sob irradiação rapidamente retornam à forma fechada incolor (Esquema 1). Ao fim de algum tempo estabelece-se um equilíbrio entre os processos de coloração e descoloração designado por equilíbrio 
fotoestacionário e caracterizado por uma constância na intensidade de cor. Quando a fonte de radiação é removida ocorre unicamente o processo de descoloração térmica das formas abertas cuja cinética pode ser facilmente seguida utilizando um espectrofotómetro de UV-VIS 9 .

Apesar de terem espectros de absorção similares, as duas formas abertas não têm igual estabilidade. $\mathrm{O}$ isómero TC é o menos estável e retorna em poucos segundos à forma fechada incolor, enquanto que o isómero TT é mais estável e reverte lentamente ao estado inicial $^{10}$. Deste modo observa-se inicialmente um rápido decaimento da coloração amarela seguido de uma lenta diminuição devido à maior estabilidade do isómero TT. Esta diferença de estabilidade das formas abertas é responsável pela persistência de uma leve coloração amarela algumas horas após o fim da irradiação.

\section{SÍNTESE DE NAFTOPIRANOS}

Existem vários métodos de síntese de naftopiranos. O método mais simples e versátil envolve a reacção de naftóis com diarilprop2-in-1-óis em meio ácido ${ }^{11,12}$. O 3,3-difenil-3H-nafto[2,1-b]pirano 1 pode ser obtido no laboratório por reacção do 2-naftol com o 1,1difenilprop-2-in-1-ol, catalizada pelo ácido $p$-toluenossulfónico em clorofórmio ou tolueno (Esquema 2). A reacção é lenta à temperatura ambiente mas fica praticamente concluída após aquecimento da solução em refluxo durante $30 \mathrm{~min}$. Após um simples tratamento é possível isolar o produto por cristalização.
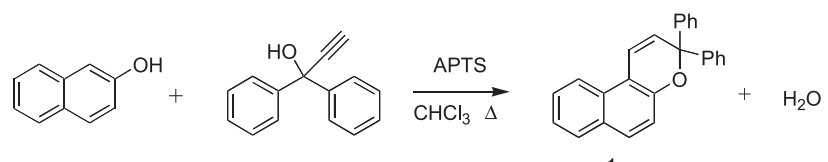

Esquema 2. Síntese do naftopirano 1

O mecanismo desta reacção é complexo e envolve a formação inicial de um éter arílico, não isolável nestas condições, que imediatamente sofre um rearranjo de Claisen [3,3]-sigmatrópico seguido de enolização, migração de hidrogénio [1,5] e finalmente uma electrociclização (Esquema 3) ${ }^{13}$. Este mecanismo é um bom exemplo de uma reacção que se processa em diversas etapas.

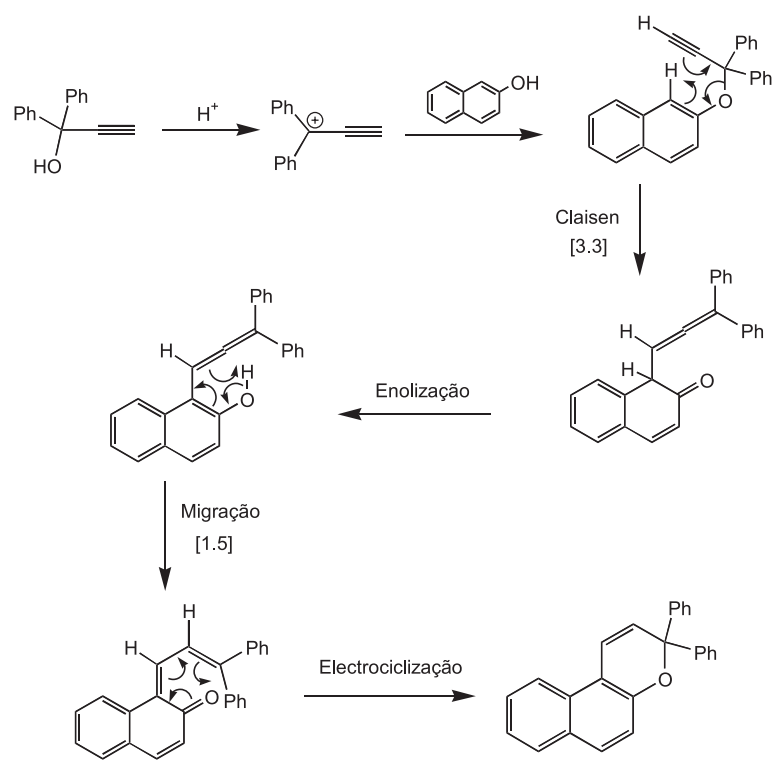

Esquema 3. Mecanismo da reacção do 2-naftol com o 1,1-difenilprop-2-in1-ol em meio ácido
Para além de serem relativamente fáceis de sintetizar, estes compostos apresentam uma elevada resistência à fotodegradação (são capazes de efectuar um grande número de processos de coloração-descoloração sem significativa degradação) e possibilitam a obtenção de cores em todo o espectro visível, mediante determinadas alterações estruturais. Devido a estas características, os naftopiranos têm sido intensamente usados nos últimos anos na produção de lentes oftálmicas que escurecem com luz solar ${ }^{3,14,15}$.

\section{MEDIÇÃO DAS PROPRIEDADES FOTOCRÓMICAS}

As propriedades fotocrómicas dos naftopiranos, sob condições de irradiação contínua, são normalmente determinadas num espectrofotómetro UV-VIS. A célula, contendo uma solução de concentração conhecida do composto a analisar é colocada no espectrofotómetro e as leituras são efectuadas ao mesmo tempo que a célula é irradiada com luz UV proveniente de uma lâmpada de Xénon, conduzida até à célula por uma fibra óptica ${ }^{6}$. A temperatura, intensidade de agitação da solução e a potência da radiação que chega à célula são factores que têm de ser controlados para se obter resultados reprodutíveis.

Neste trabalho pretende-se medir o comprimento de onda de absorção máxima das formas abertas e fechada do naftopirano 1 e estimar as cinéticas de descoloração das formas abertas coradas, mas usando meios muito mais simples. Como fonte de radiação UV pode ser utilizado um simples retroprojector de transparências ou, no caso de estarmos perante um dia de sol, a simples exposição directa à luz solar. Dado que os naftopiranos mais simples têm cinéticas de descoloração relativamente lentas, a célula contendo a solução pode ser exposta à radiação UV fora do espectrofotómetro e de seguida colocada no respectivo compartimento para se medir a absorvância. O tempo que, neste caso, medeia entre o fim da exposição e o início das medições (alguns segundos) não é significativo para os objectivos deste trabalho. Do mesmo modo, para se estimar os parâmetros acima indicados não é fundamental controlar a potência da radiação e a temperatura da solução na célula. Esta experiência destina-se a alunos universitários e pode ser efectuada em disciplinas de química orgânica. O trabalho laboratorial é fácil de realizar e visualmente apelativo uma vez que envolve uma mudança de cor reversível e permite fazer uma ponte entre a síntese orgânica e o estudo das propriedades físico-químicas dos materiais ${ }^{16}$.

\section{PARTE EXPERIMENTAL}

O 2-naftol, 1,1-difenilprop-2-in-1-ol e o ácido p-toluenosulfónico foram obtidos comercialmente (Sigma-Aldrich). O espectro no Infra-Vermelho foi registrado num espectrofotómetro Unicam FTIR Research Series utilizando uma pastilha de $\mathrm{KBr}$. O espectro de ${ }^{1} \mathrm{H}$ RMN foi obtido num aparelho Varian Unity Plus a $300 \mathrm{MHz}$ usando TMS como padrão interno e $\mathrm{CDCl}_{3}$ como solvente. Os espectros no Ultravioleta-Visível foram obtidos num espectrofotómetro Varian Cary-50 na região entre 300 e 700 nm.

\section{Síntese do 3,3-difenil-3H-nafto[2,1-b]pirano 1}

Uma solução de 2-naftol (144 mg, 1,0 mmol), 1,1-difenilprop2-in-1-ol (250 mg, 1,2 mmol), ácido $p$-toluenossulfónico (10 mg) e $\mathrm{CHCl}_{3}(20 \mathrm{~mL})$ foi aquecida em refluxo durante $30 \mathrm{~min}$. A solução inicialmente amarela vai escurecendo progressivamente obtendo-se no final uma solução verde escura. Após arrefecimento, cerca de metade do solvente foi removido num evaporador rotativo e a solução restante (cerca de $10 \mathrm{~mL}$ ) transferida para um copo de 
$50 \mathrm{~mL}$. $30 \mathrm{~mL}$ de metanol foram adicionados obtendo-se uma solução amarelada. O copo foi colocado num banho de água/gelo durante $20 \mathrm{~min}$. Neste período o naftopirano 1 cristaliza sob a forma de agulhas brancas. A suspensão assim obtida foi filtrada e os cristais secos ao ar. Obtiveram-se $205 \mathrm{mg}$ de produto. Rendimento: $61 \%$. Ponto de fusão: $158^{\circ} \mathrm{C}$. IV $\left(\mathrm{cm}^{-1}\right): 3060,1635,1588,1490$, $1460,1241,1215,1008 .{ }^{1} \mathrm{H}$ RMN: $\delta 7,96(\mathrm{~d}, J=8,4 \mathrm{~Hz}, 1 \mathrm{H}), 7,72$ (d, $J=8,1 \mathrm{~Hz}, 1 \mathrm{H}), 7,66$ (d, $J=8,7 \mathrm{~Hz}, 1 \mathrm{H}), 7,52-7,22(\mathrm{~m}, 5 \mathrm{H}), 7,36-$ 7,23 (m, 8H), 7,21 (d, J=9,0 Hz, 1H), 6,28 (d, J=10,0 Hz, 1H).

\section{Preparação de soluções}

25,00 $\mathrm{mL}$ de uma solução $10^{-3} \mathrm{M}$ do naftopirano $\mathbf{1}$ em tolueno foram preparados dissolvendo $8,4 \mathrm{mg}$ de $1 \mathrm{em} 5 \mathrm{~mL}$ de tolueno num copo de $20 \mathrm{~mL}$. Esta solução foi transferida quantitativamente para um balão volumétrico de $25,00 \mathrm{~mL}$ e o volume deste foi completado com tolueno. A partir desta solução preparou-se, por diluição, 10,00 mL de uma solução $10^{-4} \mathrm{M}$. Estas soluções podem ser guardadas à temperatura ambiente, no escuro, durante semanas sem significativa degradação.

\section{Determinação dos comprimentos de onda de absorção máxima das formas fechada e aberta}

Cerca $3 \mathrm{~mL}$ de tolueno foram transferidos para uma célula de quartzo (10 mm de percurso óptico) e o espectro de absorção do tolueno medido entre 300-700 nm num espectrofotómetro de UVVIS. Esta solução constitui o branco. A célula foi depois cheia com a solução $10^{-4} \mathrm{M}$ do naftopirano 1 e colocada de novo no espectrofotómetro de UV-VIS. O espectro de absorção da forma fechada foi traçado entre 300-700 nm registando-se depois o comprimento de onda dos máximos de absorção da forma fechada. A célula foi de seguida retirada do espectrofotómetro e aproximada, com agitação contínua, da lente de um retroprojector de transparências durante 15 a $30 \mathrm{~s}$. Neste período de tempo desenvolve-se uma intensa coloração amarela (como alternativa a célula pode ser exposta directamente à luz solar durante o mesmo tempo). A célula foi rapidamente colocada no espectrofotómetro de UV-VIS e o espectro de absorção da forma aberta medido entre $300-700 \mathrm{~nm}$. Registou-se de seguida o comprimento de onda dos máximos de absorção da forma aberta.

\section{Determinação da cinética de descoloração das formas abertas}

A célula foi lavada com tolueno e cheia de novo com a solução $10^{-4} \mathrm{M}$ do naftopirano 1 . Programou-se o espectrofotómetro para ler a absorvância da solução ao comprimento de onda do máximo de absorção da forma aberta, $426 \mathrm{~nm}$, durante $10 \mathrm{~min}$, e acertou-se o zero com a solução do naftopirano $10^{-4} \mathrm{M}$ não irradiada. A célula foi de seguida retirada do espectrofotómetro, aproximada, com agitação contínua, da lente de um retroprojector de transparências durante 15 a $30 \mathrm{~s}$, ou exposta directamente à luz solar, e colocada de novo no espectrofotómetro. Registou-se a absorvância da solução a $426 \mathrm{~nm}$, em função do tempo. Os dados foram gravados num ficheiro ASCII e transferidos para uma folha de cálculo.

\section{RESULTADOS E DISCUSSÃO}

A síntese do 3,3-difenil-3H-nafto[2,1-b]pirano a partir do 2-naftol e do 1,1-difenilprop-2-in-1-ol é um trabalho laboratorial de química orgânica rápido e de fácil execução. No final o produto da reacção, obtido por cristalização, está suficientemente puro para se prosseguir os estudos do seu comportamento fotocrómico. O espectro de
UV-VIS obtido utilizando uma solução $10^{-4} \mathrm{M}$ (Figura 1) indica que a molécula apresenta bandas de absorção intensas na região 300-400 $\mathrm{nm}$ sendo portanto possível activá-la por irradiação com luz na região do UV próximo. Este composto não apresenta bandas de absorção no visível e portanto as suas soluções são incolores.

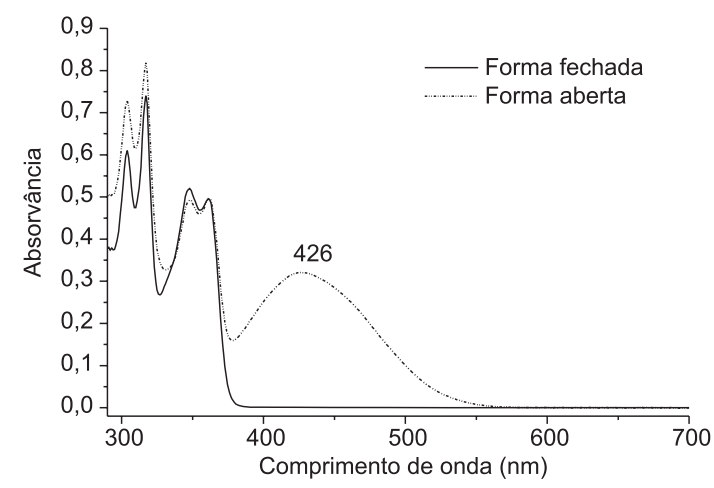

Figura 1. Espectro de absorção na região Ultravioleta-Visível das formas abertas (- - ) e fechada (_) do naftopirano $\mathbf{1}$

A exposição desta solução a radiação solar ou à luz proveniente de uma lâmpada de um retroprojector permite activar as moléculas deste composto transformando-se parte delas nas respectivas formas abertas. O espectro de UV-VIS da solução após irradiação (Figura 1) mostra o aparecimento de uma banda larga com um máximo de absorção a $426 \mathrm{~nm}$ responsável pela coloração amarela. Sem a fonte de radiação as formas abertas coloridas e instáveis revertem para a forma fechada incolor observando-se com o tempo o progressivo desaparecimento da coloração amarela (Figura 2).

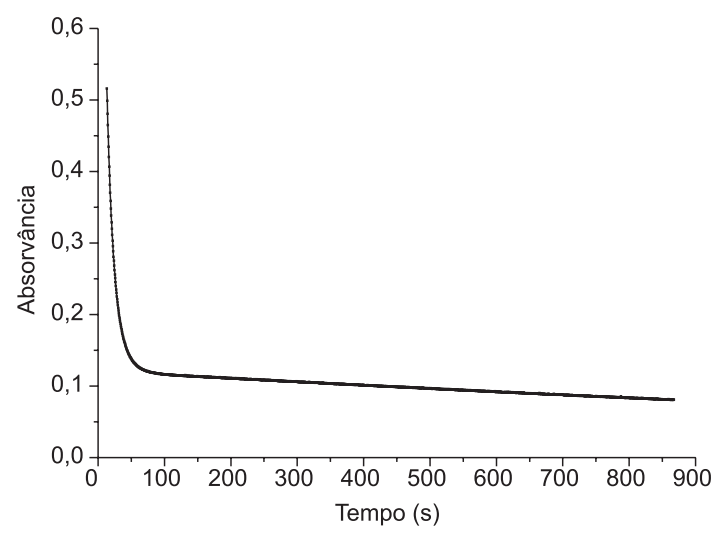

Figura 2. Cinética de descoloração do naftopirano 1

Dado que durante a irradiação com luz UV se formam duas espécies coradas, a absorvância da solução, A, durante a descoloração é dada por

$\mathrm{A}=\mathrm{B}_{1} \mathrm{e}^{-\mathrm{k}_{1} \mathrm{t}}+\mathrm{B}_{2} \mathrm{e}^{-\mathrm{k}_{2} \mathrm{t}}$

onde $\mathrm{k}_{1}$ e $\mathrm{k}_{2}$ são as constantes de descoloração das duas formas abertas (TT e TC) e $\mathrm{B}_{1}$ e $\mathrm{B}_{2}$ as absorvâncias de cada uma destas espécies no equilíbrio fotoestacionário, imediatamente antes da fonte de irradiação ser removida $(\mathrm{t}=0)$. Dado que para o naftopirano $\mathbf{1}$ as duas constantes cinéticas de descoloração são muito diferentes $\left(k_{1}>>k_{2}\right)$ elas podem ser facilmente estimadas através de um simples tratamento matemático: nos primeiros segundos após a 
remoção da fonte de irradiação, o segundo termo, $\mathrm{B}_{2} \mathrm{e}^{-\mathrm{k}_{2} \mathrm{t}}$, não vai variar significativamente dado que $\mathrm{k}_{2}$ é muito pequeno. Assim, o logaritmo da absorvância da solução nos primeiros segundos deve variar linearmente com o tempo:

$\mathrm{t} \rightarrow 0 \quad \mathrm{~B}_{2} \mathrm{e}^{-\mathrm{k}_{2} \mathrm{t}} \sim \mathrm{C}^{\mathrm{te}} \Rightarrow \ln \mathrm{A}=-\mathrm{k}_{1} \mathrm{t}+\mathrm{c}$

Para valores elevados de t, o $1^{\circ}$ termo, $B_{1} e^{-k_{1} t}$, aproxima-se de zero (dado que $\mathrm{k}_{1}$ é elevado) pelo que $\ln (\mathrm{A})$ deve variar linearmente com o tempo:

$\mathrm{t} \rightarrow \infty \mathrm{B}_{1} \mathrm{e}^{-k_{1} \mathrm{t}} \sim 0 \Rightarrow \ln \mathrm{A}=-\mathrm{k}_{2} \mathrm{t}+\mathrm{C}$

A representação do logaritmo da absorvância, ln (A), em função do tempo (t) mostra uma curva com duas assímptotas (Figura 3). O declive da curva $\ln (\mathrm{A})$ versus tempo, quando $\mathrm{t} \rightarrow 0$ permite obter $-\mathrm{k}_{1}$ enquanto que o declive da curva quando $t \rightarrow \infty$ nos dá $-k_{2}$.

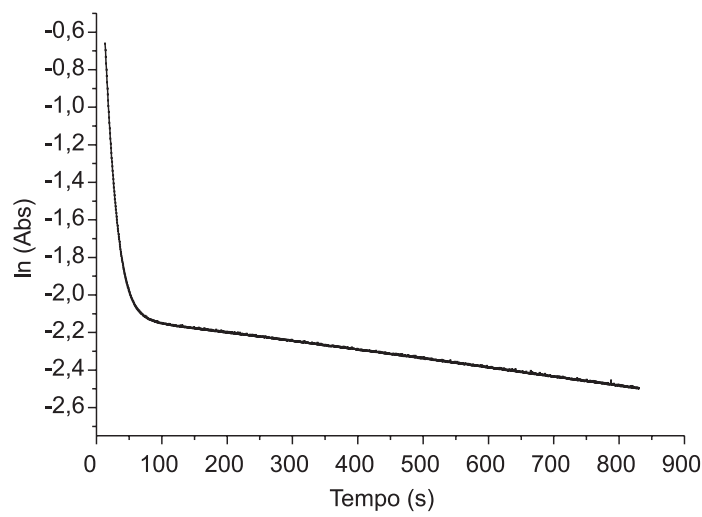

Figura 3. Representação de $\ln (A b s)$ em função do tempo $t$

Os valores de $0,06 \mathrm{~s}^{-1}$ e $0,0005 \mathrm{~s}^{-1}$ foram obtidos respectivamente para as constantes $\mathrm{k}_{1}$ e $\mathrm{k}_{2}$ o que está de acordo com um sistema constituído por uma espécie mais instável, TC, de decaimento rápido $\left(\mathrm{k}_{1}=0,06 \mathrm{~s}^{-1}\right)$ e outra mais estável, TT, de decaimento muito lento $\left(\mathrm{k}_{2}=0,0005 \mathrm{~s}^{-1}\right){ }^{6}$. Estes resultados são consistentes com as observações experimentais de que a coloração amarela decai muito rapidamente nos primeiros $30 \mathrm{~s}$ e depois muito lentamente nos min seguintes.

\section{CONCLUSÕES}

Este trabalho experimental permite aos alunos sintetizar um composto fotocrómico e estudar o seu comportamento cinético sob condições de irradiação contínua. A síntese é rápida e fácil sendo o produto isolado por cristalização. A experiência fotoquímica é visualmente apelativa dado que expondo uma solução deste composto, durante alguns segundos, a uma fonte de radiação UV se observa uma variação reversível de cor. O tratamento matemático da cinética de descoloração permite obter as constantes cinéticas de cada uma das formas abertas responsáveis pela coloração amarela. A reversibilidade do fenómeno fotocrómico, bem demonstrado na exposição do composto directamente à luz solar durante alguns segundos, é particularmente atractiva para os alunos.

\section{REFERÊNCIAS}

1. Bertelson, R. C. Em Photochromism; Brown, G. H. ed., John Wiley: New York, 1971, cap 2.

2. van Gemert, B. Em Organic Photochromic and Thermochromic Compounds; Crano, J. C.; Guglielmetti, R. J., eds.; Plenum Press: NewYork, 1999, vol. 1, cap. 3

3. Higgins, S.; Chemistry in Britain 2003, 39, 26.

4. Crano, J.; Flood, T.; Knowles, D.; Kumar, A.; van Gemert, B.; Pure Appl. Chem. 1996, 68, 1395.

5. van Gemert, B.; Mol. Cryst. Liq. Cryst. 2000, 344, 57.

6. Coelho, P. J.; Salvador, M. A.; Oliveira, M. M.; Carvalho, L. M.; Tetrahedron 2004, 60, 2593

7. Kumar, A.; van Gemert, B.; Knowles, D. B.; Mol. Cryst. Liq. Cryst. 2000, $344,217$.

8. Coelho, P. J.; Carvalho, L. M.; Abrantes, S.; Oliveira, M. M.; OliveiraCampos, A. M. F.; Samat, A.; Guglielmetti, R.; Tetrahedron 2002, 58, 9505.

9. Salvador, M. A.; Coelho, P. J.; Burrows, H. D.; Oliveira, M. M.; Carvalho, L. M.; Helv. Chim. Acta 2004, 87, 1400.

10. Delbaere, S.; Micheau, J.-C.; Vermeersch, G.; J. Org. Chem. 2003, 68, 8968.

11. Iwai, I.; Ide, J.; Chem. Pharm. Bull. 1963, 11, 1042.

12. Gabbutt, C. D.; Heron, B. M.; Instone, A. C.; Thomas, D. A.; Partington, S. M.; Hursthouse, M. B.; Gelbrich, T.; Eur. J. Org. Chem. 2003, 1220

13. Zindely J.; Schimd, H.; Helv. Chim. Acta 1968, 51, 1511.

14. van Gemert, B.; U.S. Patent 5,645,767 1997.

15. Petrosvskaia, O. G.; Kumar, A.; U.S. Patent 0071247 A1 2003.

16. Prypsztejn, H. E.; Negri, R. M.; J. Chem. Educ. 2001, 78, 645. 